\title{
REVISIONES
}

\section{Variación genética en Nothofagus (subgénero Nothofagus)}

\author{
Genetic variation in Nothofagus (subgenus Nothofagus)
}

\author{
Andrea C Premoli ${ }^{\text {a*, }}$ M Cristina Acosta ${ }^{\mathrm{b}}$, Paula Mathiasen ${ }^{\mathrm{a}}$, Claudio Donoso Z ${ }^{\mathrm{c}}$ \\ *Autor de correspondencia: ${ }^{a}$ Universidad Nacional del Comahue-CONICET, Quintral 1250, 8400 Bariloche, Argentina, \\ phone/fax: + 542944 422111, andrea.premoli@gmail.com \\ b Universidad Nacional de Córdoba-CONICET, Instituto Multidisciplinario de Biología Vegetal (IMBIV), Córdoba, Argentina. \\ c Universidad Austral de Chile, Valdivia, Chile.
}

\begin{abstract}
SUMMARY
Subgenus Nothofagus consists of five woody species that are present in diverse forest associations of temperate forests of Argentina and Chile. Given the variable conditions of the physical environment, structural and functional intraspecific variation with genetic basis is expected. The study of the gene pool of multiple populations of the different species at the regional scale using conserved nuclear and cytoplasm markers resolved the phylogenetic relationships within the subgenus and reconstructed their biogeographic history in Patagonia, respectively. Nuclear ITS sequences showed that $N$. pumilio diverged earlier and that $N$. antarctica is sister to the monophyletic group containing the three evergreens ( $N$. betuloides, $N$. dombeyi, and $N$. nitida). Phylogeographic analyses based on sequences of chloroplast DNA allowed the reconstruction of ancient features of the Oligocene-Miocene landscape of Patagonia and showed paleohybridizations. The contemporaneous signal yielded by isozyme polymorphisms contributed to the analysis of spatial variation patterns such as the effect of the geographic range, the formation of clines and ecotypes, the genetic consequences of natural disturbances in relation to the predominant regeneration mode (sprouter and nonsprouter), and hybridization. Analysis on distinct habitat types inhabited by $N$. antarctica and experimental studies under common gardens and reciprocal transplants of $N$. pumilio at contrasting elevations showed genetic and plastic responses in ecophysiological and morphological characters. Taxa within subgenus Nothofagus are ancient lineages that have developed adaptations throughout their evolutionary history and therefore they may have the potential to respond to changes in climate.
\end{abstract}

Key words: genetic variation, geographic variation, Nothofagus, phylogeography, subgenus Nothofagus.

\section{RESUMEN}

El subgénero Nothofagus consiste de cinco especies leñosas que están presentes en diversas asociaciones forestales de los bosques templados de Argentina y Chile. Dadas las variaciones del medio físico se esperan variaciones intraespecíficas estructurales y funcionales con base genética. El análisis del acervo genético de múltiples poblaciones de las distintas especies a escala regional, mediante marcadores nucleares y citoplasmáticos con baja tasa de mutación, permitió resolver las relaciones filogenéticas y reconstruir su historia biogeográfica en Patagonia, respectivamente. Secuencias nucleares ITS mostraron que N. pumilio divergió tempranamente y que $N$. antarctica resultó ser hermana del grupo monofilético conteniendo las tres especies siempreverdes ( $N$. betuloides, $N$. dombeyi y N. nitida). Análisis filogeográficos mediante secuencias de ADN del cloroplasto reconstruyeron rasgos antiguos del paisaje del Oligoceno-Mioceno de Patagonia y develaron la existencia de paleohibridaciones. La señal contemporánea provista por polimorfismos isoenzimáticos contribuyó al análisis de patrones espaciales de variación como el efecto del rango geográfico, la formación de clines y ecotipos, las consecuencias genéticas de los disturbios naturales en relación con el modo predominante de regeneración (rebrotante y no-rebrotante) y la hibridación. Estudios en distintos ambientes habitados por N. antarctica y experimentales en jardín común y trasplantes recíprocos de $N$. pumilio en alturas contrastantes, mostraron que caracteres ecofisiológicos y morfológicos de los individuos son el resultado de selección natural y plasticidad. Las especies del subgénero Nothofagus son linajes antiguos que han desarrollado adaptaciones a lo largo de su historia evolutiva y, por lo tanto, tendrían el potencial de responder a cambios en el clima.

Palabras clave: filogeografía, Nothofagus, subgénero Nothofagus, variación genética, variación geográfica. 


\section{INTRODUCCIÓN}

Las especies con un extenso rango de distribución, generalmente, ocupan una amplia gama de ambientes y muestran variación geográfica en distintos caracteres. Esta variación fenotípica puede resultar de la expresión variable de determinados genotipos en distintos ambientes (plasticidad fenotípica) o de la adaptación, lo cual implica la selección de distintos genotipos bajo condiciones ambientales heterogéneas. Además de la selección natural, otras fuerzas evolutivas como el reducido tamaño poblacional (deriva genética) y el aislamiento espacial y temporal afectan los polimorfismos genéticos en las poblaciones naturales. Por lo tanto, especies de amplio rango permiten analizar los factores ambientales e históricos que han modelado la variación genética de sus poblaciones.

El rango de distribución geográfica juntamente con el sistema reproductivo son los caracteres de historia de vida de las especies que mejor explican los niveles de diversidad genética en las poblaciones de plantas (Hamrick y Godt 1989). Esto se debe a que las especies de amplia distribución y con fecundación cruzada usualmente consisten de poblaciones grandes y que mantienen altas tasas de flujo génico entre ellas. Asimismo, el medio físico cambiante genera presiones divergentes de selección (Linhart y Grant 1996). Se espera que especies con amplias distribuciones que habitan ambientes variables mantengan altos niveles de diversidad genética.

Los paisajes de bosque de Patagonia han sido modelados por distintos procesos ocurridos a lo largo de la historia geológica de los Andes del sur. Estos ambientes de montaña consisten de numerosos valles, cuyas laderas con distinta orientación y pendiente imponen condiciones variables sobre las especies que las habitan. Además, la influencia del Océano Pacífico en el oeste con innumerables fiordos y archipiélagos y el efecto sombra sobre las laderas orientales de los Andes resultan en ambientes contrastantes. Así, Claudio Donoso (1987) postuló que "cualquier especie que se desarrolle en un rango de distribución más o menos amplio en este territorio, se va a encontrar con este medioambiente en extremo variado". Esto fue motivo de un estudio fundacional en el género Nothofagus Blume el cual describió la variación intraespecífica de atributos seminales e hibridación interespecífica a base de caracteres morfológicos de las especies que crecen en Chile (Donoso 1987). Dicho trabajo además planteó hipótesis para futuros estudios. Por un lado se propuso que las especies mostrarían plasticidad fenotípica a lo largo de gradientes altitudinales continuos, mientras que con la latitud las diferencias entre las poblaciones tendrían un fuerte componente genético, además del ambiental, por efecto de la distancia y posibles discontinuidades geográficas. En el presente trabajo se analizan dichas hipótesis en especies del subgénero Nothofagus. Las especies de este subgénero poseen las mayores distribuciones en el sur de Sudamérica y proveen el experimento natural para analizar el grado y el tipo de variación geográfica.

\section{LAS ESPECIES DEL SUBGÉNERO NOTHOFAGUS}

El subgénero Nothofagus consiste de cinco especies. Dos de éstas son deciduas o de hoja caduca conocidas comúnmente como ñire ( $N$. antarctica (G. Forst.) Oerst.) y lenga (N. pumilio (Poepp. et Endl.) Krasser) y las otras tres son siempreverdes: coihue (N. dombeyi (Mirb.) Oerst.), coihue de Magallanes o guindo ( $N$. betuloides (Mirb.) Oerst.) y coihue de Chiloé (N. nitida (Phil.) Krasser). Los patrones de variación intraespecíficos de las especies del subgénero Nothofagus tanto fenotípicos como genotípicos son complejos ya que estas especies poseen amplias distribuciones y habitan ambientes heterogéneos. Este es el caso de los dos taxa deciduos cuyas distribuciones coinciden con el rango total latitudinal del bosque templado austral. Presentan variación significativa en distintos rasgos estructurales y funcionales. Nothofagus antarctica presenta variación morfológica discreta (ecotípica) asociada a distintos ambientes (biotopos). Se han sugerido distintos morfotipos (Ramírez et al. 1985, Premoli 1991) que podrían considerarse ecotipos resultantes de presiones de selección relativamente recientes tendientes a la especiación (Romero 1986a). Por otro lado, N. pumilio es la especie que domina los ambientes montanos y de altas latitudes donde comúnmente forma bosques puros. Sobre las laderas de montaña, esta entidad muestra variación continua (clinal) en rasgos morfológicos de los individuos hasta alcanzar el límite de la vegetación arbórea (Premoli 2004). De las tres especies siempreverdes, $N$. betuloides y $N$. dombeyi son de amplia distribución. Nothofagus betuloides es el elemento siempreverde característico de los bosques subantárticos, mientras que hacia el norte se lo encuentra en bosques templados de altura y en sitios anegados o de turbera y ñadis. Nothofagus dombeyi ocurre hacia el norte de los $43^{\circ} \mathrm{S}$ en bosques templados y húmedos de mediana altitud. Por último, $N$. nitida presenta un rango latitudinal menor que las restantes especies del subgénero y está restringido a los bosques templados y húmedos de mediana latitud sobre las vertientes occidentales de los Andes. Las especies del subgénero son consideradas tolerantes al frío, principalmente la siempreverde $N$. betuloides (Premoli 1994) y las deciduas $N$. antarctica y N. pumilio (Alberdi 1987).

Los miembros del subgénero comparten la dispersión del polen anemófila y una dispersión limitada de las semillas que ocurre en la cercanía del árbol semillero. Por lo tanto, se podría postular que diferencias genéticas entre poblaciones geográficamente cercanas y sin barreras aparentes al intercambio genético sólo podrán generarse por acción de la selección natural diversificadora en casos que supere los efectos homogeneizadores del flujo génico. De la misma manera, los patrones de establecimiento de semillas afectan las características genéticas dentro de los rodales de Nothofagus. Estos rodales pueden considerarse sistemas de no-equilibrio (Veblen y Donoso 1987); es decir, son perturbados frecuentemente por disturbios naturales que afectan su demografía. Las consecuencias 
genéticas dependerán de la escala espacial del disturbio (de grano fino a escala local o grueso a mayor escala espacial) y del modo de regeneración preponderante (no-rebrotante y por lo tanto semilla-dependiente como $N$. dombeyi versus rebrotante como $N$. antarctica). Así la regeneración de no-rebrotantes en claros abiertos en el bosque por caída de árboles de $N$. dombeyi promoverá el establecimiento local de semillas y la formación de una estructuración demográfica y genética significativa (Premoli y Kitzberger 2005). En tanto que la recolonización de $N$. dombeyi luego de eventos devastadores como fuego, avalanchas, caídas masivas por viento, tendrá lugar a través de propágulas producidas por escasos árboles remanentes resultando en rodales coetáneos y genéticamente homogéneos (Premoli y Kitzberger 2005). Por el contrario, la regeneración de especies rebrotantes preservará la estructura demográfica y genética previa a la perturbación. Los rodales postfuego de $N$. antarctica resultaron genéticamente diversos a escala de rodal (1 ha) y estructurados espacialmente ya que aún individuos cercanos consistían de distintos genotipos (genets) (Premoli y Steinke 2008). Mientras disturbios devastadores como el fuego producen una homogeneización genética en $N$. dombeyi (no-rebrotante), el establecimiento ocasional por semilla reforzado por rebrote conservan una estructura genética significativa en $N$. antarctica. Por lo tanto, las consecuencias genéticas de los disturbios a nivel de rodal dependerán de la escala del mismo y del modo de regeneración de las especies.

\section{DIVERSIDAD GENÉTICA}

El efecto del rango de distribución geográfica fue analizado sobre los niveles de variación genética en Nothofagus. Se espera que especies de amplio rango mantengan elevado polimorfismo genético en comparación con sus congéneres de distribución geográfica restringida. Utilizando loci nucleares isoenzimáticos, se encontró que las especies siempreverdes ampliamente distribuidas, $N$. betu- loides y N. dombeyi, mostraron mayor diversidad isoenzimática que la restringida $N$. nitida (Premoli 1997). Por otro lado, las especies deciduas de amplia distribución, $N$. antarctica y $N$. pumilio, tuvieron mayor y menor diversidad que las siempreverdes de amplio rango, respectivamente (cuadro 1). Además, todas las especies de amplia distribución mostraron una divergencia interpoblacional significativa $\left(\mathrm{F}_{\mathrm{ST}}\right)$ excepto la de menor rango $N$. nitida.

La elevada diversidad en las especies de amplio rango como las siempreverdes $N$. betuloides y $N$. dombeyi se podría explicar con base en sus grandes poblaciones (Premoli 1997), mientras que en la decidua $N$. antarctica se relacionaría con su gran tolerancia ecológica y su capacidad de rebrote luego de disturbios que permite preservar la estructura genética de las poblaciones (Acosta et al. 2012). Por el contrario, si bien $N$. pumilio posee el mismo rango latitudinal que $N$. antarctica, el mismo es relativamente discontinuo particularmente en su distribución norte donde ocupa ambientes de altura, habitando elevaciones más bajas solamente hacia el sur. La especificidad de hábitat de $N$. pumilio, restringida ecológicamente a ambientes fríos, como así también la competencia con otras especies, limitan eventualmente los sitios que puede habitar. Esto sumado a restricciones al establecimiento de propágulas particularmente hacia el límite altitudinal superior que resulta en poblaciones de menor tamaño efectivo (Premoli 2003). Asimismo, el estudio de 85 poblaciones de todo el rango de distribución con base en secuencias de tres regiones de ADN del cloroplasto (ADNc) mostró que aquellas del norte habrían sufrido severos cuellos de botella históricos (Mathiasen y Premoli 2010). La combinación de estos factores habría erosionado la diversidad genética de $N$. pumilio. Por otro lado, $N$. nitida con menor rango latitudinal que el resto tuvo una menor diversidad genética aunque sin barreras aparentes al flujo génico interpoblacional (valores de $\mathrm{F}_{\mathrm{ST}}$ no estadísticamente distintos de cero) (cuadro 1) por la relativa continuidad de sus poblaciones habitando ambientes similares. Esto apoya la hipótesis que el rango

Cuadro 1. Medidas promedio de diversidad genética total $\left(\mathrm{H}_{\mathrm{T}}\right)$ e intrapoblacional $\left(\mathrm{H}_{\mathrm{S}}\right)$ para cada especie, endogamia intrapoblacional promedio $\left(\mathrm{F}_{\mathrm{IS}}\right)$ y grado de divergencia interpoblacional $\left(\mathrm{F}_{\mathrm{ST}}\right)$ para todas las especies del subgénero Nothofagus en base a loci nucleares isoenzimáticos polimórficos $\left(\mathrm{N}_{\mathrm{L}}\right)$.

Average metrics for total $\left(\mathrm{H}_{\mathrm{T}}\right)$ and within-population genetic diversity $\left(\mathrm{H}_{\mathrm{S}}\right)$ for each species, average within-population inbreeding $\left(\mathrm{F}_{\mathrm{IS}}\right)$, and degree of among-population divergence $\left(\mathrm{F}_{\mathrm{ST}}\right)$ for all species of subgenus Nothofagus based on nuclear isozyme polymorphic loci $\left(\mathrm{N}_{\mathrm{L}}\right) . *$ significantly different from zero values.

\begin{tabular}{lcccccl}
\hline \multicolumn{1}{c}{ Especie, nombre común } & $\mathrm{H}_{\mathrm{T}}$ & $\mathrm{H}_{\mathrm{S}}$ & $\mathrm{F}_{\mathrm{IS}}$ & $\mathrm{F}_{\mathrm{ST}}$ & $\mathrm{N}_{\mathrm{L}}$ & Referencias \\
\hline N. nitida, coihue de Chiloé & 0,047 & 0,042 & $0,121^{*}$ & 0,131 & 4 & Premoli 1997 (datos) \\
N. dombeyi, coihue & 0,264 & 0,233 & $0,198^{*}$ & $0,266^{*}$ & 6 & Premoli y Kitzberger 2005 \\
N. betuloides, guindo & 0,150 & 0,116 & $0,131^{*}$ & $0,269^{*}$ & 6 & Premoli 1997 (datos) \\
$N$. pumilio, lenga & 0,089 & 0,072 & $0,296^{*}$ & $0,200^{*}$ & 7 & Mathiasen 2010, Mathiasen y Premoli 2010 \\
$N$. antarctica, ñire & 0,276 & 0,235 & $0,283^{*}$ & $0,182^{*}$ & 7 & Premoli y Steinke 2008, Steinke et al. 2008 \\
\hline
\end{tabular}

* Valores significativamente distintos de cero. 
sería un buen predictor de la diversidad genética de las poblaciones (Hamrick y Godt 1989, Premoli 1997).

El modo de regeneración predominante por rebrote o semilla también afectará los niveles y distribución de la variación genética a escala regional. Utilizando marcadores isoenzimáticos en 20 pares de poblaciones simpátricas se encontró que $N$. antarctica posee mayor diversidad genética a lo largo de su rango de distribución latitudinal que la no-rebrotante $N$. pumilio. Esto fue explicado en relación a la persistencia por rebrote de $N$. antarctica en poblaciones de mayor tamaño. Juntamente con una mayor plasticidad fenotípica resulta en mayor resiliencia de $N$. antarctica a condiciones cambiantes de Patagonia (Acosta et al. 2012). Así poblaciones de N. antarctica sufrieron en menor grado los efectos de la deriva genética que tiende a erosionar la variación genética en poblaciones pequeñas y relativamente aisladas de $N$. pumilio especialmente hacia el norte de su distribución.

\section{FLUJO GÉNICO INTRA- E INTER-ESPECÍFICO}

Una manera de estudiar el tipo de sistema reproductivo es estimar las tasas de fecundación cruzada a base de pruebas de progenie (genotipos de semillas mantenidas en familias, es decir, provenientes de un árbol semillero dado). También se puede analizar la reducción en la heterocigosis media de la población por efecto de la endogamia. En las especies siempreverdes se encontró una elevada tasa de fecundación cruzada relativa $(t \geq 0,873)$ (Premoli 1996a). Coincidentemente, cruzamientos experimentales en $N$. dombeyi y $N$. nitida mostraron que serían altamente auto-incompatibles (Riveros et al. 1995). Sin embargo, la falta de diferencias significativas en las frecuencias alélicas de la progenie y los presuntos progenitores sugiere que las especies siempreverdes ajustarían a un modelo de sistema reproductivo mixto producto de la auto y alogamia (Premoli 1996a). Por otro lado, en todas las especies se midió una deficiencia en la heterocigosis con estimaciones de la endogamia $\left(\mathrm{F}_{\mathrm{IS}}\right)$ significativamente distintas de cero (cuadro 1). Esto indica que cierto grado de autofecundación o de endogamia biparental debido a la cruza de individuos genéticamente similares puede ocurrir en las distintas especies del subgénero Nothofagus.

La fecundación preponderantemente cruzada puede ocurrir además entre distintas especies a través de la hibridación. Las especies del subgénero comparten el mismo tipo de polen. Por lo tanto, la abundancia dispar de polen puede resultar en competencia por polen y en la formación de híbridos interespecíficos. Ya Donoso (1987) daba cuenta de hibridación entre las especies siempreverdes en base a caracteres foliares, seminales y de los frutos (Donoso y Atienza 1983, 1984). La hibridación entre $N$. nitida y $N$. betuloides y posible introgresión en la dirección de esta última fue confirmada mediante análisis isoenzimáticos (Premoli 1994, 1996a). La mayor similitud isoenzimática y morfológica entre $N$. dombeyi y $N$. betuloides fue inter- pretada como hibridación o divergencia reciente (Premoli 1996b, 1997). Además, tal como fuera sugerido por Donoso (1987) se demostró la hibridación entre $N$. antarctica y $N$. pumilio en distintas localidades a lo largo de la distribución de las especies puras. Análisis morfológicos y moleculares en áreas de Tierra del Fuego (Premoli 2004) y en el norte de la Patagonia (Quiroga et al. 2005) confirmaron la existencia de hibridación y posible introgresión hacia el acervo genético de $N$. antarctica. Se documentó también la ocurrencia de hibridación entre la especie decidua $N$. antarctica y la siempreverde $N$. dombeyi (Stecconi et al. 2004). Esto muestra que existirían barreras reproductivas incompletas dentro del subgénero aún entre especies con diferente hábito de las hojas (caduca y siempreverde).

Las especies del subgénero Nothofagus no solamente tienen el potencial de hibridar entre sí actualmente, sino que lo han hecho en el pasado. La concordancia en la distribución de polimorfismos de ADN del cloroplasto de las cinco especies, permitió determinar que poseen una historia evolutiva compartida que data del Paleógeno la cual ha sido mantenida por ciclos de hibridación e introgresión (Acosta y Premoli 2010, Premoli et al. 2012). Sin embargo, las especies son claramente morfológica y ecológicamente distinguibles entre sí como así también a base de polimorfismos moleculares mediante marcadores nucleares de ITS (Acosta y Premoli 2010). Esto podría interpretarse como que la hibridación puede haber jugado un rol fundamental favoreciendo la dispersión y recolonización de las especies a través del flujo de polen interespecífico y la permanencia de las especies del subgénero Nothofagus en ambientes como los de la Patagonia que han sido recurrentemente afectados por disturbios. Sin embargo, las presiones diferenciales producto de los ambientes heterogéneos de Patagonia (Donoso 1987) han mantenido la integridad de las especies de Nothofagus de amplio rango por selección ecológica.

\section{HUELLA PALEÓGENA}

La variación en el subgénero Nothofagus es difícilmente separable del componente histórico. Es decir, las poblaciones de las especies actuales no solamente reflejan procesos de adaptación sino también eventos históricos que dejaron su huella en el acervo genético de las especies. Sin embargo, el impacto de procesos ocurridos en el pasado dependerá de las características autoecológicas de las especies. Se plantea la hipótesis que especies tolerantes al frío como las del subgénero Nothofagus habrían podido sobrevivir in situ a los períodos glaciarios. La supervivencia en áreas potencialmente libres de hielo y, por lo tanto, de refugio de bosque habría conservado la estructura genética ancestral. De esta forma, marcadores nucleares codominantes podrían develar restricciones contemporáneas al flujo génico mientras que el uso de marcadores genéticos con baja tasa de mutación como de ADN del cloroplasto permitiría reconstruir eventos que precedieron a las glaciaciones más antiguas de Patagonia que tuvieron lugar hace 
aproximadamente cinco a siete millones de años (Rabassa et al. 2005).

El subgénero Nothofagus posee un registro de polen fósil que data desde el Terciario (Dettmann et al. 1990). En Sudamérica, probablemente el subgénero ya estaba presente como pequeñas poblaciones en el Eoceno de Patagonia (Romero 1986b). Existen registros del tipo de polen que caracteriza el subgénero Nothofagus (fusca-b) en al menos dos localidades; hacia el sur en la Formación Ligorio Márquez (ca. $47^{\circ} \mathrm{S}, 72^{\circ} \mathrm{O}$ ) (Okuda et al. 2006) con una edad datada de 47,6 \pm 0,78 Ma (Yabe et al., 2006) y hacia el norte en río Pichileufú $\left(\mathrm{ca} .41^{\circ} \mathrm{S}, 71^{\circ} \mathrm{O}\right)^{1}$ con una edad de 47,46 \pm 0,05 Ma (Wilf et al. 2005). Esto da cuenta de la evolución in situ del subgénero en Patagonia.

Si bien las relaciones filogenéticas de los subgéneros fueron postuladas inicialmente a base de los tipos de polen (Hill y Read 1991) y confirmadas luego mediante análisis moleculares [Lophozonia (Turcz.) Krasser [Fuscospora R. S. Hill y J. Read [Nothofagus, Brassospora W. R. Philipson y M. N. Philipson]]] (por ejemplo, Manos 1997), las relaciones entre las especies dentro de los subgéneros permanecían aún sin esclarecerse completamente. El análisis de poblaciones y especies del subgénero Nothofagus presentes en 10 localidades a lo largo de su distribución en Patagonia a base de secuencias de tres regiones de $\mathrm{ADNc}$ y la región nuclear ITS mostró que solamente los marcadores nucleares permiten reconstruir las relaciones evolutivas entre las especies del subgénero (Acosta y Premoli 2010). Este análisis filogenético permitió elucidar que $N$. pumilio divergió primeramente del resto siendo $N$. antarctica la especie hermana del clado monofilético conteniendo a las especies siempreverdes ( $N$. dombeyi, $N$. nitida y $N$. betuloides) dentro del cual $N$. nitida habría divergido más tempranamente (Acosta y Premoli 2010) (figura 1). La datación molecular del árbol filogenético tanto con marcadores nucleares como del cloroplasto utilizando fósiles para la calibración mostró que el subgénero Nothofagus presenta una antigüedad de al menos $50 \mathrm{Ma}$ (Premoli et al. 2012) La datación indicó además que la divergencia de $N$. pumilio, $N$. antarctica y $N$. nitida ocurrieron hace 22,13 y 6 millones de años, respectivamente, mientras que la de $N$. betuloides y $N$. dombeyi habría tenido lugar hace aproximadamente 1 millón de años (Premoli et al. 2012). La divergencia de $N$. pumilio fue contemporánea a la de $N$. alessandrii Esp. (ruil) del subgénero Fuscospora la cual ha sido considerada hasta el momento como representante de la condición ancestral del género Nothofagus lo que estaría mostrando la evolución temprana de las especies actuales del género Nothofagus en el Oligoceno de Sudamérica. Es posible, que estos eventos de especiación en distintos subgéneros ocurrieron posteriormente al enfriamiento del clima que pasó de un régimen tropicalsubtropical a templado durante el Oligoceno. Mientras que

\footnotetext{
${ }^{1} \mathrm{~W}$ Volkheimer, IANIGLA/CRICYT, Mendoza, Argentina. Comunicación personal.
}

especies mesotérmicas como $N$. alessandri divergieron y quedaron restringidas al norte de la Patagonia, la microtérmica $N$. pumilio probablemente evolucionó desarrollando la tolerancia al frío siendo exitosa en colonizar ambientes de altura y de altas latitudes con su resultante amplia distribución actual. La decidua $N$. antarctica y las siempreverdes probablemente evolucionaron al establecerse tendencias climáticas de mayor aridez. La primera desarrollando adaptaciones a distintos ambientes mientras que las siempreverdes quedando restringidas a bosques húmedos. En particular, $N$. nitida, especie siempreverde del subgénero Nothofagus de menor rango restringida a las vertientes occidentales de los Andes, probablemente evolucionó una vez que se elevó la Cordillera de Los Andes durante el Mioceno (Premoli et al. 2012). El parentesco más cercano entre las siempreverdes $N$. dombeyi y $N$. betuloides fue sugerido con anterioridad a base del análisis poblacional utilizando marcadores nucleares isoenzimáticos (Premoli 1994, 1996a). Además, estudios aplicando técnicas morfométricas sobre plantas de las especies siempreverdes cultivadas bajo condiciones homogéneas (jardín común) sugirieron una relación evolutiva más cercana entre $N$. dombeyi y $N$. betuloides que entre éstas y $N$. nitida (Premoli 1994, 1996b). Si bien la divergencia de N. dombeyi y $N$. betuloides puede relacionarse con las oscilaciones climáticas acaecidas desde el Plioceno (Romero 1986a) la de $N$. nitida y las restantes especies actuales del subgénero Nothofagus probablemente precedió dichos eventos (Premoli 1997, Premoli et al. 2012).

Las secuencias de ADNc no reflejaron las relaciones filogenéticas existentes entre las especies sino que resultaron estar asociadas a regiones geográficas. Es decir, en un área determinada las especies presentes comparten las mismas secuencias de ADNc (Acosta y Premoli 2010). Esto significa que las especies no pueden ser identificadas a base de marcadores de ADNc, probablemente debido al fenómeno de hibridación. Las especies del subgénero Nothofagus comparten el mismo tipo de polen y se ha sugerido que existirían barreras reproductivas débiles entre ellas (Veblen et al. 1996). Además, se han documentado casos de hibridación entre distintos pares de especies del subgénero (Donoso y Atienza 1983, 1984, Donoso 1987, Premoli 1994, 1996a, Stecconi et al. 2004, Quiroga et al. 2005). Por lo tanto, en casos en que el polen exógamo es escaso y si la densidad de flores interespecíficas supera a las intraespecíficas, la hibridación y retrocruzas con los parentales podría ocurrir como fuera documentado en otras especies vegetales (Cruzan et al. 1994). Este mecanismo ocurre tal que la especie con tamaños poblacionales reducidos actúa como receptora de polen de la especie que se encuentra en mayoría dando como resultado progenie híbrida. Si continúan los cruzamientos de los híbridos con el parental más abundante mediante el proceso denominado introgresión (retrocruzas recurrentes) se producirán individuos cuya constitución del núcleo será aquella de la especie dadora de polen, mientras que conservará el citoplasma 


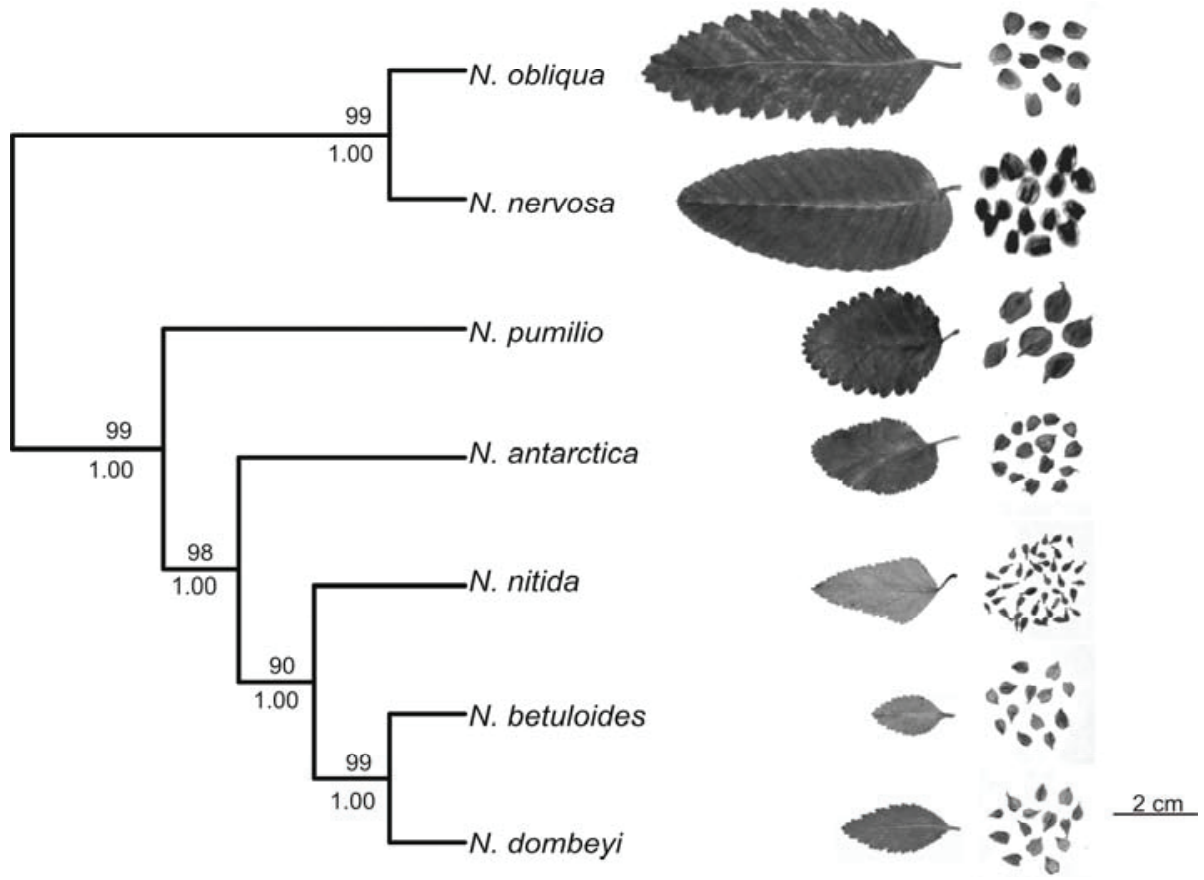

Figura 1. Relaciones filogenéticas de las especies del subgénero Nothofagus con base en secuencias de ADN nuclear de la región ITS (Acosta y Premoli 2010). A modo ilustrativo se incluye su morfología foliar y características seminales. Las especies $N$. obliqua y $N$. nervosa del subgénero Lophozonia fueron utilizadas como grupo externo. Números por encima y debajo de las ramas indican valores de remuestreo con reposición del análisis de máxima parsimonia y probabilidades posteriores de la inferencia Bayesiana, respectivamente.

Phylogenetic relationships within subgenus Nothofagus based on DNA sequences of the ITS nuclear region (Acosta and Premoli 2010). As an illustration, leaf and seed morphology of each species are included. Members of subgenus Lophozonia, N. obliqua, and N. nervosa were used as outgroups. Numbers above and below branches indicate bootstrap values for maximum parsimony and posterior probabilities from Bayesian inference analyses, respectively.

y, por consiguiente, los cloroplastos de la especie receptora, fenómeno conocido como captura del cloroplasto. La hibridación y posterior introgresión podría ocurrir luego de perturbaciones en los que escasos individuos remanentes sobreviven al disturbio y/o durante la expansión poblacional (ver figura 4 en Acosta y Premoli 2010). Se podría postular la hipótesis que esto habría ocurrido a lo largo de la historia evolutiva del subgénero Nothofagus en Patagonia y que habría resultado en una estructura de ADNc fuertemente controlada por la geografía. Sin embargo, además de las secuencias compartidas entre especies simpátricas se encontraron secuencias especie-específicas para las regiones de $\mathrm{ADNc}$ analizadas (por ejemplo, haplotipo $\mathrm{H} 3 \mathrm{de}$ $N$. antarctica y $\mathrm{H} 8$ de $N$. betuloides, figura $1 \mathrm{~B}$ de Acosta y Premoli 2010) y para otros marcadores de ADNc estudiados mediante RFLP-PCR (Soliani et al. 2011).

Las variantes de ADNc de las especies del subgénero Nothofagus mostraron una distribución geográfica concordante en las distintas especies las cuales estuvieron asociadas a la latitud. El análisis filogenético de las secuencias de ADNc arrojó dos grupos principales distribuidos al norte y sur de los $43^{\circ}$ de latitud sur. Tal concordancia a lo largo de toda la región de los bosques de Patagonia, es la más extensa mapeada hasta el momento. La distribución de variantes de ADN de organelas (del cloroplasto en plantas y mitocondria en animales) consiste de regiones que no recombinan y son heredadas de manera uniparental. Por lo tanto, los cambios poseen una tasa de evolución lenta y son acumulados como secuencias ordenadas de eventos mutacionales que permiten mapear eventos del pasado. Este es el objetivo de la disciplina conocida como filogeografía (Avise et al. 1987). Discontinuidades filogeográficas, es decir, patrones discontinuos en la distribución geográfica de variantes genéticas, pueden indicar la presencia de barreras geográficas al flujo génico a través de las semillas. Si además dichas discontinuidades son concordantes entre distintos taxones o entre distintos marcadores genéticos dará idea de la existencia y persistencia de dichas barreras (Avise et al. 1987). La datación de tal divergencia en dos grupos monofiléticos indicó la existencia de una barrera geográfica Paleógena de $30 \mathrm{Ma}$ a los $43^{\circ} \mathrm{S}$ asociada a una transgresión marina ocurrida desde el Pacífico (Premoli et al. 2012). La existencia de áreas emergentes en Patagonia como el macizo Norpatagónico y el del Deseado al sur, habrían permitido la persistencia de miembros del subgénero Nothofagus y la conservación de tales polimorfismos 
en el ADNc a través del tiempo (Mathiasen y Premoli 2010). Otra divergencia antigua de unos 20 Ma fue encontrada más hacia el norte a los $40^{\circ} \mathrm{S}$. Similares divergencias en regiones del ADNc analizadas mediante RFLP-PCR fueron reportadas en $N$. nervosa (Phil.) Krasser (raulí) al norte (Marchelli et al. 1998) y en N. antarctica y N. pumilio al sur (Pastorino et al. 2009, Soliani et al. 2011) que fueron interpretadas como producto del aislamiento en distintos refugios glaciarios. Si bien las glaciaciones pueden haber afectado la estructura genética de las poblaciones, se requiere el uso de secuencias de ADN conservado, análisis filogeográficos y la combinación con marcadores nucleares para poder desentrañar la señal contemporánea (i.e., glaciaciones) de la antigua (i.e., rasgos paleogeográficos del paisaje) (Mathiasen y Premoli 2010). Una prueba de la existencia de una barrera antigua a los $43^{\circ} \mathrm{S}$ lo demuestra la presencia de dos grupos genéticamente diferenciados en la especie de amplia distribución Podocarpus nubigena (Quiroga y Premoli 2010). Por lo tanto, la estructura genética de linajes filogenéticamente independientes da cuenta de una barrera geográfica al flujo génico generalizada a latitudes medias de Patagonia. La presencia de esta discontinuidad refuerza la idea que el agua habría actuado como una barrera a la dispersión en especies de Nothofagus y otros grupos de plantas. De ser así, los patrones de diversidad de especies podrían también haber sido afectados por dicha barrera. Así por ejemplo, distribuciones disyuntas en Patagonia para algunos linajes de plantas han sido mayormente explicadas en relación con la historia glaciaria Neógena (Villagrán e Hinojosa 2005). Sin embargo, en el caso de especies tolerantes al frío, dichas disyunciones latitudinales podrían reflejar eventos más antiguos. Es decir, linajes de amplia distribución que sufrieron disyunciones debido a la barrera producida por la transgresión del Pacífico entre el Oligoceno y el Mioceno.

\section{LAS GLACIACIONES DEL NEÓGENO}

El registro de polen fósil y el mapeo de depósitos glaciares permitieron analizar las respuestas de la vegetación durante los cambios del clima en los últimos millones de años en Patagonia. Se postuló que la vegetación habría subsistido en áreas libres de hielo en particular hacia bajas latitudes en el norte de la Patagonia. Este escenario, similar al planteado para especies de plantas del Hemisferio Norte, fue cuestionado por Markgraf et al. (1995) que propuso la hipótesis que las características oceánicas, juntamente con la heterogeneidad ambiental del sur de Sudamérica, habría favorecido la persistencia de la vegetación en sitios microclimatológicamente favorables durante el último máximo glaciar (UMG). Sin embargo, el uso del registro polínico en el subgénero Nothofagus es problemático ya que las distintas especies poseen el mismo tipo de polen. Por otro lado, la baja abundancia de polen en un sitio determinado podría deberse a la persistencia local o al arribo reciente por dispersión a distancia las cuales tendrían con- secuencias genéticas diferentes y difíciles de desentrañar. Mediante el uso de marcadores nucleares isoenzimáticos se analizó la hipótesis de la supervivencia in situ a lo largo de la distribución actual de las especies leñosas tolerantes al frío. En particular, si las poblaciones ubicadas hacia el extremo austral (frío) de distribución fueran producto de la dispersión a distancia, se esperaría que fueran genéticamente derivadas de las del norte. Además, deberían estar empobrecidas genéticamente debido a que la dispersión a distancia generalmente involucra cuellos de botella ocurridos durante la migración postglaciaria. El análisis preliminar a lo largo de la distribución de $N$. pumilio (Premoli 1998) y que fuera confirmado por estudios posteriores a base de un mayor número de poblaciones (Mathiasen y Premoli 2010) mostró que las poblaciones del extremo sur poseen niveles de diversidad genética comparables a los de las poblaciones del norte. Además, presentaron variantes genéticas únicas las que habrían tendido a perderse por efecto fundador durante la dispersión a distancia. Estos resultados, juntamente con evidencia genética en la conífera tolerante al frío alerce (Fitzroya cupressoides (Molina) Johnston), llevó a plantear por primera vez la hipótesis de refugios múltiples sensu Premoli (1998) (Premoli et al. 2000). Estudios detallados a lo largo de la latitud en N. pumilio postularon que las diferencias en la historia glaciaria en el norte y el sur de la distribución de la especie afectaron diferencialmente la estructura genética poblacional. Inesperadamente, las poblaciones del sur tuvieron mayor heterocigosidad y riqueza alélica $\left(\mathrm{H}_{\mathrm{O}}=0,55\right.$ y $\left.\mathrm{A}_{\mathrm{R}}=1,06\right)$ que las del norte $\left(\mathrm{H}_{\mathrm{O}}=0,32\right.$ y $\left.\mathrm{A}_{\mathrm{R}}=0,43\right)$. Sin embargo, las del norte mantuvieron mayor flujo génico y por lo tanto mostraron menor divergencia poblacional $\left(\mathrm{F}_{\mathrm{ST}}=0,059\right.$, Intervalo de Confianza del $\left.95 \%\left(\mathrm{IC}_{95 \%}\right)=0,019-0,159\right)$ que las del sur $\left(\mathrm{F}_{\mathrm{ST}}=0,217, \mathrm{IC}_{95 \%}=0,166-0,243\right)$ (Mathiasen y Premoli 2010). La mayor diversidad en el sur, principalmente en una especie como $N$. pumilio con limitada dispersión de semillas, sugiere que pudo subsistir in situ en poblaciones grandes en áreas probablemente extensas que permanecieron aisladas entre sí. Por el contrario, en el norte de Patagonia la glaciación consistió principalmente de glaciares de valle donde la mayor cantidad de áreas libres de hielo facilitaron la permanencia de $N$. pumilio en numerosas y pequeñas poblaciones (Mathiasen y Premoli 2010); si bien sufrieron los efectos de la endogamia, mantuvieron continuo flujo génico entre ellas. La tesis de la supervivencia de $N$. pumilio a altas latitudes durante los períodos fríos fue apoyada por evidencia independiente de modelaje de nicho ecológico, que permite proyectar la distribución de las especies en el pasado a base de modelos climáticos. Juntamente con información molecular, se postuló que las poblaciones actuales de $N$. pumilio de Tierra del Fuego serían el resultado de refugios de bosque que se habrían ubicado sobre la costa Atlántica consecuencia de una disminución del nivel del mar y corrimiento hacia el este de la línea de costa a partir de los cuales se habría producido la recolonización posglaciaria (Premoli 
et al. 2010). La secuenciación de regiones no recombinantes de ADNc confirmaron la supervivencia local en múltiples sitios a lo largo de la distribución actual de las especies del subgénero Nothofagus conservando la señal genética antigua (Premoli et al. 2012).

\section{VARIACIÓN EN AMBIENTES ESPACIAL Y TEMPORALMENTE HETEROGÉNEOS}

Las especies deciduas $N$. antarctica y $N$. pumilio presentan el mayor grado de variación fenotípica del subgénero Nothofagus siendo en la primera de tipo discontinua (ecotípica) asociada a distintos ambientes y continua (clinal) en el gradiente altitudinal en la segunda. Si bien trabajos previos describieron la variación fenotípica en condiciones naturales para distintos caracteres en N. antarctica (Ramírez et al. 1985, Premoli 1991) y N. pumilio (por ejemplo, Rusch 1993, Cuevas 2000) se planteó el interrogante acerca de si dicha variación resulta de los efectos del ambiente (plasticidad fenotípica) o si las mismas tendrían una base genética. Debido a posibles variaciones geográficas en caracteres adaptativos, estos estudios se llevaron a cabo a escala local en bosques del norte de la Patagonia a fin de controlar por potenciales efectos latitudinales que pueden resultar en diferencias adaptativas (Fajardo y Piper 2011).

El diseño experimental en $N$. antarctica consistió de pares de poblaciones simpátricas en los distintos ambientes que habita la especie. Estos fueron matorral de fondo de valle y montano de ladera y sitios de bosque y ambientes anegados (de mallín, turbera o ñadis). Sobre árboles adultos se efectuaron análisis de atributos arquitecturales de los individuos, morfológicos de las hojas y genéticos isoenzimáticos. Además, se caracterizó ambientalmente a los sitios mediante sus características geográficas y edáficas. Si bien la variación genética fue evaluada con marcadores isoenzimáticos que muestran principalmente los efectos del aislamiento y la deriva genética, estos loci neutrales podrían sufrir un efecto de "arrastre genético" por estar ligados a caracteres adaptativos. Por lo tanto, se podría plantear que los patrones de variación isoenzimáticos podrían relacionarse con los efectos de la selección natural. Las distintas poblaciones resultaron heterogéneas para rasgos morfológicos. Así, poblaciones simpátricas resultaron significativamente diferentes entre sí para la altura del fuste y el número de ramas por individuo (Steinke et al. 2008). El análisis multivariado incluyendo todos los caracteres mostró que las poblaciones de bosque y de altura resultaron ser las más diferenciadas (Steinke et al. 2008), mientras que las de matorral y turbera no presentaron diferencias significativas. Los marcadores moleculares arrojaron una alta diversidad genética para todas las poblaciones (heterocigosis esperada $\mathrm{H}_{\mathrm{E}}>0,18$ ) y a pesar de tratarse de poblaciones geográficamente cercanas poseen un aislamiento genético entre ellas moderado pero significativo $\left(\mathrm{F}_{\mathrm{ST}}=18 \%\right.$ ) (Steinke et al. 2008). Es posible entonces que la gran diversidad fenotípica en $N$. antarctica sea producto de la combinación de plasticidad fenotípica y variación genética.

Para separar los efectos ambientales de los genéticos en $N$. pumilio, se combinaron estudios moleculares con experimentos en jardín común y de transplantes recíprocos entre procedencias con condiciones altitudinales contrastantes para analizar respuestas adaptativas, es decir, el ajuste diferencial de determinadas variantes genotípicas a condiciones ambientales heterogéneas. El análisis mediante electroforesis isoenzimática de poblaciones ubicadas en distintos pisos altitudinales mostraron que $N$. pumilio posee baja diversidad genética $\left(\mathrm{H}_{\mathrm{E}}<0,051\right)$ y que el grado de variación genética (es decir, el polimorfismo) disminuye con la altura (Premoli 2003). La escasa diversidad genética con la altura puede relacionarse con cuellos de botella para el reclutamiento en altura (Cuevas 2000), mientras que el aumento del establecimiento de plántulas en baja altitud mantendría mayores tamaños efectivos y explicaría la mayor diversidad genética encontrada en estas poblaciones. Se encontró además que el grado de divergencia entre poblaciones de distintas alturas de un mismo cerro resultó significativo $\left(\mathrm{F}_{\mathrm{ST}} \leq 13 \%\right)$ mientras que entre distintos cerros fue solamente del $2 \%$. La diferenciación genética encontrada entre poblaciones separadas por escasos cientos de metros a lo largo de gradientes altitudinales se relaciona con las marcadas diferencias fenológicas con la altura. Estudios de campo mostraron que existe un retardo de aproximadamente seis días en la iniciación de la floración por cada 100 metros de diferencia en altitud (Rusch 1993). Las flores femeninas de N. pumilio son receptivas por una semana; además, las flores masculinas son efímeras ya que tienden a caer por efecto de los fuertes vientos de la primavera. Por lo tanto, se puede plantear que existirían barreras reproductivas efectivas aún entre poblaciones cercanas habitando un mismo cerro, mientras que poblaciones ubicadas en pisos altitudinales similares en distintos cordones montañosos pueden mantener mayor flujo génico efectivo entre ellas (a través del polen) debido a la sincronía en la fenología, aun estando distantes entre sí. La diferenciación genética entre distintos pisos altitudinales sería reforzada por los efectos de la dispersión limitada de las semillas de $N$. pumilio que raramente supera los $100 \mathrm{~m}$, siendo la mayoría dispersadas en la cercanía $(<20 \mathrm{~m}$ ) del árbol madre (Cuevas 2000). Los experimentos en jardín común de plántulas de $N$. pumilio provenientes de alturas contrastantes permitieron evaluar caracteres ecofisiológicos (densidad estomática, tasa fotosintética máxima y conductancia estomática) y de crecimiento y fenología. Se encontraron diferencias significativas en la tasa fotosintética máxima siendo las de mayor altitud las que alcanzaron los mayores valores promedio $( \pm 1 \mathrm{~S})$ tanto en condiciones naturales $18,3(0,6)$ y $10,5(0,6) \mu \mathrm{mol} \mathrm{CO}_{2}$ $\mathrm{m}^{-2} \mathrm{~s}^{-1}$ para mayor y menor altitud, respectivamente como en el jardín común $7,7(0,4)$ y $6,3(0,4) \mu \mathrm{mol} \mathrm{CO}_{2} \mathrm{~m}^{-2} \mathrm{~s}^{-1}$ 
para mayor y menor altitud, respectivamente (Premoli y Brewer 2007). La densidad estomática y la conductancia presentaron mayores valores en las plantas de mayor altitud bajo condiciones naturales mientras que las mismas no presentaron diferencias significativas en el jardín común, lo que indicaría que las diferencias observadas en campo son producto del ambiente (Premoli y Brewer 2007). En el norte de la Patagonia, $N$. pumilio habita climas de régimen Mediterráneo con sequías estivales. Para una especie decidua como $N$. pumilio sería ventajoso poseer respuestas plásticas en relación con el uso del agua y así poder utilizar el agua disponible a lo largo de la estación de crecimiento. Por otro lado, las diferencias en la fotosíntesis resultaron tener un control genético significativo que estaría relacionado con el tipo de sustrato que habita $N$. pumilio. Se trata de suelos volcánicos pobres en nitrógeno y donde se favorecería su conservación a partir de la formación de hojas ricas en compuestos secundarios como la lignina y nitrógeno foliar sobre todo a mayor altitud (ver figura 1, Premoli y Mathiasen 2011). Esto a su vez se traduciría en una mayor inversión en el aparato fotosintético y mayores tasas de asimilación. El monitoreo del crecimiento y fenología de las plantas cultivadas en jardín común, permitió determinar que existen diferencias genéticas en caracteres morfológicos de las hojas, arquitecturales de los individuos y fenológicas. Las plantas provenientes de menores alturas mostraron un mayor crecimiento del eje principal del tallo, mayor tamaño foliar y un retraso en la fenología de la brotación al inicio de la estación de crecimiento. Además se pudo determinar que las plantas provenientes de mayor altitud tuvieron una mayor tasa y ángulos de ramificación que las de menor altitud (Premoli et al. 2007). Esto refuerza la idea que las plantas de $N$. pumilio poseen diferencias genéticas que son mantenidas por la selección natural bajo condiciones contrastantes. Por otro lado, los transplantes recíprocos mostraron a base de caracteres de crecimiento y morfología foliar mayor capacidad de respuesta a las condiciones del transplante por parte de las plantas de menor altitud (Premoli y Mathiasen 2011). Este resultado juntamente con la mayor variación genética neutral encontrada en estas últimas le conferiría ventajas adaptativas ante la relajación de presiones de selección en altura (Premoli y Mathiasen 2011).

\section{CONCLUSIONES}

El subgénero Nothofagus consiste de linajes antiguos que evolucionaron y que han subsistido in situ desde el Oligoceno de Patagonia. Reconstrucciones filogeográficas utilizando ADN del cloroplasto y dataciones mediante reloj molecular determinaron que dichos linajes sufrieron disyunciones por barreras a la dispersión debido a entradas marinas desde el Pacífico durante el Oligoceno-Mioceno. Estas disyunciones resultaron geográficamente concordantes para las cinco especies dando cuenta de paleohibridaciones. Además, muestra que el agua es un impedimento para la dis- persión en Nothofagus y apoya la hipótesis de divergencia por vicarianza. Los polimorfismos de marcadores nucleares biparentales muestran una señal contemporánea y sugieren que especies del subgénero Nothofagus tolerantes al frío pudieron sobrevivir durante los períodos fríos en múltiples áreas libres de hielo a lo largo de sus distribuciones actuales habiendo ocurrido en el caso de $N$. pumilio en áreas tan australes como Tierra del Fuego. Esto contrasta con lo encontrado para especies leñosas del Hemisferio Norte que se habrían extinguido en latitudes altas habiendo recolonizado luego del retiro de los hielos desde refugios ubicados en zonas templado-cálidas. Bajo escenarios de calentamiento se podría postular que las características oceánicas del extremo austral de Sudamérica y la presencia de la cordillera de los Andes favorecerían movimientos locales altitudinales y longitudinales a lo largo de las distribuciones actuales de la especies sin mayores corrimientos latitudinales.

Las especies de amplio rango que habitan diversos ambientes con capacidad de rebrote como $N$. antarctica o que consisten de poblaciones grandes como $N$. dombeyi y $N$. betuloides poseen elevada diversidad genética en sus poblaciones. Si bien la especie montana $N$. pumilio posee una distribución latitudinal similar a $N$. antarctica, su reducida diversidad genética sería producto de su estrecho rango ecológico, el aislamiento en ambientes de montaña y cuellos de botella sufridos a lo largo de su historia evolutiva. Por otro lado, la reducida diversidad genética encontrada en la especie de menor rango latitudinal $N$. nitida muestra que el rango puede ser un buen predictor de los niveles de variación genética en poblaciones.

Las poblaciones de las especies del subgénero Nothofagus varían espacial y temporalmente y esta variación posee base genética para algunos caracteres funcionales. Esto ocurre en respuesta a presiones diferenciales de selección en distintos ambientes como en $N$. antarctica y debido al aislamiento temporal por el desacople en la fenología aún entre poblaciones separadas por escasos cientos de metros en $N$. pumilio. Se podría predecir que ante cambios climáticos la ecológicamente tolerante $N$. antarctica probablemente ajustará a cambios en el clima merced a su gran polimorfismo genético y plasticidad fenotípica. Si bien se postulan ascensos altitudinales para los bosques de N. pumilio restringidos a ambientes de montaña, es de esperar que los mismos ocurran a partir de migraciones desde los pisos altitudinales inferiores caracterizados por mayor capacidad reproductiva y diversidad genética que se traduciría en un mayor potencial de respuesta ante condiciones cambiantes.

Las poblaciones de las especies del subgénero Nothofagus son frecuentemente perturbadas. Dependiendo de la escala espacial del disturbio y el modo de regeneración predominante como rebrotante o no-rebrotante será la estructura genética resultante. Mientras que la diversidad genética es conservada por rebrote en rodales postdisturbio de $N$. antarctica, la misma se correlaciona con la disponibilidad y éxito reproductivo de árboles semilleros sobrevivientes al disturbio en las no-rebrotantes como 
N. dombeyi. Deben monitorearse sitios postdisturbio, como por ejemplo luego de incendios, ya que los mismos suelen utilizarse para el pastoreo del ganado, a fin de evitar la erosión genética en dichos rodales.

Los patrones de variación genética en Nothofagus ocurren a escala (1) regional consecuencia de factores históricos, (2) de paisaje entre poblaciones habitando distintos ambientes y (3) poblacional dentro de rodales producto del modo de regeneración. Esto resulta de combinar estudios a campo y experimentales utilizando distintos marcadores moleculares con caracteres cuantitativos juntamente con información ecofisiológica y demográfica. Este trabajo mostró los avances alcanzados en el análisis de la variación intraespecífica en Nothofagus desde la publicación en 1987 del trabajo de Donoso. Más estudios se requieren que analicen patrones de variación geográfica a distintas escalas espaciales y temporales y para distintos caracteres en especies sudamericanas de los subgéneros Fuscospora y Lophozonia.

\section{AGRADECIMIENTOS}

Agradecemos los comentarios de los revisores sobre la primera versión del manuscrito. Financiamiento: Universidad Nacional del Comahue 04/B152. Las autoras son miembros del CONICET.

\section{REFERENCIAS}

Acosta MC, AC Premoli. 2010. Evidence of chloroplast capture in South American Nothofagus (subgenus Nothofagus, Nothofagaceae). Molecular Phylogenetics and Evolution 54: 235-242.

Acosta MC, P Mathiasen, AC Premoli. 2012. Predominant regeneration strategy results in species-specific genetic patterns in sympatric Nothofagus s.s. congeners (Nothofagaceae). Australian Journal of Botany 60(4): 319-327 DOI: 10.1071/ BT11277.

Alberdi M. 1987. Ecofisiología de especies chilenas del género Nothofagus. Bosque 8(2): 77-84.

Avise JC, J Arnold, R Martin Ball, E Bermingham, T Lamb, JE Neigel, CA Reeb, NC Saunders. 1987. Intraspecific phylogeography: the mitochondrial DNA bridge between population genetics and systematics. Annual Review of Ecology and Systematics 18: 489-522.

Cruzan MB, JL Hamrik, ML Arnold, BD Bennett. 1994. Mating system variation in hybridizing irises: effects of phenology and floral densities on family outcrossing rates. Heredity 72: 95-105.

Cuevas JG. 2000. Tree recruitment at the Nothofagus pumilio alpine timberline in Tierra del Fuego, Chile. Journal of Ecology 88: 840-855.

Dettmann ME, DT Pocknall, EJ Romero, M del C Zamaloa. 1990. Nothofagidites Erdtman ex Potonie 1960: a catalogue of species with notes on the paleogeographic distribution of Nothofagus Bl. (southern beech). New Zealand Geological Survey Palaentological Bulletin 60: 1-79.

Donoso C. 1987. Variación natural en especies de Nothofagus en Chile. Bosque 8(2): 85-97.
Donoso C, J Atienza. 1983. Hibridación natural entre especies de Nothofagus siempreverdes en Chile. Bosque 5(1): 21-34.

Donoso C, J Atienza. 1984. Hibridación natural entre Nothofagus betuloides (Mirb.) Oerst. y Nothofagus nitida (Phill.) Krasser. Medio Ambiente (Universidad Austral de Chile) 7: 9-16.

Fajardo A, FI Piper. 2011. Intraspecific trait variation and covariation in a widespread tree species (Nothofagus pumilio) in southern Chile. New Phytologist 189: 259-271.

Hamrick JL, MJW Godt. 1989. Allozyme diversity in plant species. In Brown AHD, MT Clegg, AL Kahler, BS Weir eds. Plant population genetics, breeding and germplasm resources. Sunderland, MA, Estados Unidos. Sinauer. p 43-63.

Hill RS, J Read. 1991. A revised infrageneric classification of Nothofagus (Fagaceae). Botanical Journal of the Linnean Society 105: 37-72.

Linhart YB, MC Grant. 1996. Evolutionary significance of local genetic differentiation in plants. Annual Review of Ecology and Systematics 27: 237-277.

Manos PS. 1997. Systematics of Nothofagus (Nothofagaceae) based on rDNA spacer sequences (ITS): taxonomic congruence with morphology and plastid sequences. American Journal of Botany 84: 1137-1155.

Marchelli P, LA Gallo, F Scholz, B Ziegenhagen. 1998. Chloroplast DNA markers revealed a geographical divide across Argentinean southern beech Nothofagus nervosa (Phil.) Dim. et Mil. distribution area. Theoretical and Applied Genetics 97: 642-646.

Markgraf V, M McGlone, G Hope. 1995. Neogene paleoenvironmental and paleoclimatic change in southern temperate ecosystems - a southern perspective. Trends in Ecology and Evolution 10: 143-147.

Mathiasen P. 2010. Variación y estructura genética en Nothofagus pumilio (Poepp. et Endle.) Krasser 'lenga' a lo largo de diferentes gradientes ambientales. Tesis Doctoral, Ciencias Biológicas, Centro Regional Universitario Bariloche, Universidad Nacional del Comahue, Río Negro, Argentina. 252 p.

Mathiasen P, AC Premoli. 2010. Out in the cold: genetic variation of Nothofagus pumilio (Nothofagaceae) provides evidence for latitudinally distinct evolutionary histories in austral South America. Molecular Ecology 19: 371-385.

Okuda M, H Nishida, K Uemura, A Yabe. 2006. Paleocene/Eocene pollen assemblages from the Ligorio Márquez Formation, Central Patagonia, XI Region, Chile. In Nishida H ed. Post-Cretaceous Floristic Changes in Southern Patagonia, Chile. Tokyo, Japón. Chuo University. p. 37-43.

Pastorino MJ, P Marchelli, M Milleron, C Soliani, LA Gallo. 2009. The effect of different glaciation patterns over the current genetic structure of the southern beech Nothofagus antarctica. Genetica 136: 79-88.

Premoli AC. 1991. Morfología y capacidad germinativa en poblaciones de Nothofagus antarctica (Forster) Oerst. del noroeste andino patagónico. Bosque 12(2): 53-59.

Premoli AC. 1994. Variación genética, morfológica y ecofisiológica en especies de Nothofagus geográficamente restringida y ampliamente distribuidas del sur de Sudamérica. Tesis Doctoral, Departamento de Biología, Universidad de Colorado, Boulder, Estados Unidos. 148 p.

Premoli AC. 1996a. Allozyme polymorphisms, outcrossing rates, and hybridization of South American Nothofagus. Genetica 97: 55-64.

Premoli AC. 1996b. Leaf architecture of South American Notho- 
fagus (Nothofagaceae) using traditional and new methods in morphometrics. Botanical Journal of the Linnean Society 121: $25-40$.

Premoli AC. 1997. Genetic variation in a geographically restricted and two widespread species of South American Nothofagus. Journal of Biogeography 24: 883-892.

Premoli AC. 1998. Use of genetic markers to conserve endangered species and to design protected areas for more widespread species. In International Foundation for Science ed. Proceedings of an International Workshop on Recent Advances in Biotechnology for Tree Conservation and Management. Universidade Federal de Santa Catarina. Santa Catarina, Brazil. p. 157-171.

Premoli AC. 2003. Isozyme polymorphisms provide evidence of clinal variation with elevation in Nothofagus pumilio. Journal of Heredity 94: 218-226.

Premoli AC. 2004. Variación en Nothofagus pumilio (Poepp. et Endl.) Krasser (N. pumilio). In Donoso C, AC Premoli, L Gallo, R Iliniza eds. Variación intraespecífica en las especies arbóreas de los bosques templados de Chile y Argentina. Santiago de Chile, Chile. Editorial Universitaria. p. 145-166.

Premoli AC, C Brewer. 2007. Environmental vs. genetically driven variation in ecophysiological traits of Nothofagus pumilio from contrasting elevations. Australian Journal of Botany 55: 585-591.

Premoli AC, T Kitzberger. 2005. Regeneration mode affects spatial genetic structure of Nothofagus dombeyi forests. Molecular Ecology 14: 2319-2329.

Premoli AC, P Mathiasen. 2011. Respuestas ecofisiológicas adaptativas y plásticas en ambientes secos de montaña: Nothofagus pumilio, el árbol que acaparó los Andes australes. Ecología Austral 21: 251-269.

Premoli AC, T Kitzberger, TT Veblen. 2000. Isozyme variation and recent biogeographical history of the long-lived conifer Fitzroya cupressoides. Journal of Biogeography 27: 251260.

Premoli AC, P Mathiasen, MC Acosta, VA Ramos. 2012, Phylogeographically concordant chloroplast DNA divergence in sympatric Nothofagus s.s. How deep can it be? New Phytologist 193: 261-275.

Premoli AC, P Mathiasen, T Kitzberger. 2010. Southernmost Nothofagus trees enduring ice ages: genetic evidence and ecological niche retrodiction reveal high latitude $\left(54^{\circ} \mathrm{S}\right)$ glacial refugia. Palaeogeography, Palaeoclimatology, $\mathrm{Pa}$ laeoecology 298: 247-256.

Premoli AC, E Raffaele, P Mathiasen. 2007. Morphological and phenological differences in Nothofagus pumilio from contrasting elevations. Austral Ecology 32: 515-523.

Premoli AC, L Steinke. 2008. Genetics of sprouting: effects of long-term persistence in fire-prone ecosystems. Molecular Ecology 17: 3827-3835.

Quiroga MP, AC Premoli. 2010. Genetic structure of Podocarpus nubigena (Podocarpaceae) provides evidence of Quaternary and ancient historical events. Palaeogeography, Palaeoclimatology, Palaeoecology 285: 186-193.

Quiroga P, R Vidal Russel, AC Premoli. 2005. Evidencia morfológica e isoenzimática de hibridación natural entre Notho- fagus antarctica y N. pumilio en el noroeste Patagónico. Bosque 26(2): 25-32.

Rabassa J, AM Coronato, M Salemme. 2005. Chronology of the Late Cenozoic Patagonian glaciations and their correlation with biostratigraphic units of the Pampean region (Argentina). Journal of South American Earth Sciences 20: 81-103.

Ramírez C, M Correa, H Figueroa, J San Martin. 1985. Variación del hábito y hábitat de Nothofagus antarctica en el cento sur de Chile. Bosque 6(2): 55-73.

Riveros M, MA Parades, MT Rosas E Cárdenas, J Armesto, MTK Arroyo, B Palma. 1995 Reproductive biology in species of the genus Nothofagus. Environmental and Experimental Botany 35: 519-524.

Romero EJ. 1986a. Fossil Evidence Regarding the Evolution of Nothofagus Blume. Annals of the Missouri Botanical Garden 73: 276-283.

Romero EJ. 1986b. Paleogene phytogeography and climatology of South America. Annals of the Missouri Botanical Garden 73: 449-461.

Rusch VE .1993. Altitudinal variation in the phenology of Nothofagus pumilio in Argentina. Revista Chilena de Historia Natural 66: 131-141.

Soliani C, L Gallo, P Marchelli. 2011. Phylogeography of two hybridizing southern beeches (Nothofagus spp.) with different adaptive abilities. Tree Genetics \& Genomes DOI: 10.1007/s11295-011-0452-9.

Stecconi M, P Marchelli, J Puntieri, P Picca, LA Gallo. 2004. Natural hybridization between a deciduous (Nothofagus antarctica, Nothofagaceae) and an evergreen (N. dombeyi) forest tree species: evidence from morphological and isoenzymatic traits. Annals of Botany 94: 775-786.

Steinke L, AC Premoli, CP Souto, M Hedrén. 2008. Adaptive and neutral variation of the resprouter Nothofagus antarcti$\mathrm{ca}$ growing in distinct habitats in north-western Patagonia. Silva Fennica 42: 177-188.

Veblen TT, C Donoso. 1987. Alteración Natural y Dinámica Regenerativa de las Especies Chilenas de Nothofagus de la Región de los Lagos. Bosque 8(2): 133-142.

Veblen TT, C Donoso, T Kitzberger, AJ Rebertus. 1996. Ecology of southern Chilean and southern Argentinean Nothofagus forests. In Veblen TT, RS Hill, J Read ed. Ecology and biogeography of Nothofagus forests. New Haven, Connecticut, Estados Unidos. Yale University Press. p. 293-353.

Villagrán C, LF Hinojosa. 2005. Esquema biogeográfico de Chile. In Llorente-Bousquets J, JJ Morrone eds. Regionalización Biogeográfica en Iberoamérica y tópicos afines. Distrito Federal, México. Ediciones de la Universidad Nacional Autónoma de México, Jiménez Editores. p. 551-577.

Wilf P, KR Johnson, NR Cúneo, ME Smith, BS Singer, MA Gandolfo. 2005. Eocene plant diversity at Laguna del Hunco and Río Pichileufú, Patagonia, Argentina. American Naturalist 165: 634-650.

Yabe A, K Uemura, H Nishida. 2006. Geological notes on plant fossil localities of the Ligorio Márquez Formation, central Patagonia, Chile. In Nishida H ed. Post-Cretaceous floristic changes in southern Patagonia, Chile. Tokyo, Japón. Chuo University. p. 29-35. 
